\title{
COMBINING KNOWLEDGE SOURCES TO REORDER N-BEST SPEECH HYPOTHESIS LISTS
}

\author{
Manny Rayner ${ }^{1}$, David Carter ${ }^{1}$, Vassilios Digalakis ${ }^{2}$, Patti Price ${ }^{2}$ \\ (1) SRI International, Suite 23, Millers Yard, Cambridge CB2 1RQ, UK
}

(2) SRI International, Speech Technology and Research Laboratory, 333 Ravenswood Ave., Menlo Park, CA 94025-3493, USA

\begin{abstract}
A simple and general method is described that can combine different knowledge sources to reorder N-best lists of hypotheses produced by a speech recognizer. The method is automatically trainable, acquiring information from both positive and negative examples. In experiments, the method was tested on a 1000-utterance sample of unseen ATIS data.
\end{abstract}

\section{INTRODUCTION}

During the last few years, the previously separate fields of speech and natural language processing have moved much closer together, and it is now common to see integrated systems containing components for both speech recognition and language processing. An immediate problem is the nature of the interface between the two. A popular solution has been the $N$-best list-for example, [9]; for some $N$, the speech recognizer hands the language processor the $N$ utterance hypotheses it considers most plausible. The recognizer chooses the hypotheses on the basis of the acoustic information in the input signal and, usually, a simple language model such as a bigram grammar. The language processor brings more sophisticated linguistic knowledge sources to bear, typically some form of syntactic and/or semantic analysis, and uses them to choose the most plausible member of the N-best list. We will call an algorithm that selects a member of the N-best list a preference method. The most common preference method is to select the highest member of the list that receives a valid semantic analysis. We will refer to this as the "highest-incoverage" method. Intuitively, highest-in-coverage seems a promising idea. However, practical experience shows that it is surprisingly hard to use it to extract concrete gains. For example, a recent paper [8] concluded that the highest-incoverage candidate was in terms of the word error rate only very marginally better than the one the recognizer considered best. In view of the considerable computational overhead required to perform linguistic analysis on a large number of speech hypotheses, its worth is dubious.

In this paper, we will describe a general strategy for constructing a preference method as a near-optimal combination of a number of different knowledge sources. By a "knowledge source", we will mean any well-defined procedure that associates some potentially meaningful piece of information with a given utterance hypothesis $H$. Some examples of knowledge sources are

- The plausibility score originally assigned to $H$ by the recognizer

- The sets of surface unigrams, bigrams and trigrams present in $H$

- Whether or not $H$ receives a well-formed syntactic/semantic analysis

- If so, properties of that analysis

The methods described here were tested on a 1001-utterance unseen subset of the ATIS corpus; speech recognition was performed using SRI's DECIPHER ${ }^{T M}$ recognizer $[7,5]$, and linguistic analysis by a version of the Core Language Engine (CLE [2]). For 10-best hypothesis lists, the best method yielded proportional reductions of $13 \%$ in the word error rate and $11 \%$ in the sentence error rate; if sentence error was scored in the context of the task, the reduction was about $21 \%$. By contrast, the corresponding figures for the highestin-coverage method were a $7 \%$ reduction in word error rate, a $5 \%$ reduction in sentence error rate (strictly measured), and a $12 \%$ reduction in the sentence error rate in the context of the task.

The rest of the paper is laid out as follows. In Section 2 we describe a method that allows different knowledge sources to be merged into a near-optimal combination. Section 3 describes the experimental results in more detail. Section 4 concludes.

\section{COMBINING KNOWLEDGE SOURCES}

Different knowledge sources (KSs) can be combined. We begin by assuming the existence of a training corpus of $\mathrm{N}$-best lists produced by the recognizer, each list tagged with a "reference sentence" that determines which (if any) of the hypotheses in it was correct. We analyse each hypothesis $H$ in the corpus using a set of possible KSs, each of which associates some form of information with $H$. Information can be of two different kinds. Some KSs may directly produce a number that can be viewed as a measure of $H$ 's plausibility. Typical examples are the score the recognizer assigned to $H$, and the score for whether or not $H$ received a linguistic analysis ( 1 or 0 , respectively). More commonly, however, the KS will produce a list of one or more "linguistic items" associated with $H$, for example surface $\mathrm{N}$-grams in $H$ or the grammar rules occurring in the best linguistic analysis of $H$, if there was one. A given linguistic item $L$ is associated with a numerical score through a "discrimination function" (one function for each type of linguistic item), which summarizes the relative frequencies of occurrence of $L$ in correct and incorrect hypotheses, respectively. Discrimination functions are discussed in more detail shortly. The score assigned to $H$ 
by a KS of this kind will be the sum of the discrimination scores for all the linguistic items it finds. Thus, each KS will eventually contribute a numerical score, possibly via a discrimination function derived from an analysis of the training corpus.

The total score for each hypothesis is a weighted sum of the scores contributed by the various KSs. The final requirement is to use the training corpus a second time to compute optimal weights for the different KSs. This is an optimization problem that can be approximately solved using the method described in $[3]^{1}$.

The most interesting role in the above is played by the discrimination functions. The intent is that linguistic items that tend to occur more frequently in correct hypotheses than incorrect ones will get positive scores; those which occur more frequently in incorrect hypotheses than correct ones will get negative scores. To take an example from the ATIS domain, the trigram $a$ list of is frequently misrecognized by $\mathrm{DECIPHER}^{T M}$ as a list the. Comparing the different hypotheses for various utterances, we discover that if we have two distinct hypotheses for the same utterance, one of which is correct and the other incorrect, and the hypotheses differ by one of them containing $a$ list of while the other contains $a$ list the, then the hypothesis containing $a$ list of is nearly always the correct one. This justifies giving the trigram $a$ list of a positive score, and the trigram $a$ list the a negative one.

We now define formally the discrimination function $d_{T}$ for a given type $T$ of linguistic item. We start by defining $d_{T}$ as a function on linguistic items. As stated above, it is then extended in a natural way to a function on hypotheses by defining $d_{T}(H)$ for a hypothesis $H$ to be $\sum d_{T}(L)$, where the sum is over all the linguistic items $L$ of type $T$ associated with $H$.

$d_{T}(L)$ for a given linguistic item $L$ is computed as follows. (This is a slight generalization of the method given in [4].) The training corpus is analyzed, and each hypothesis is tagged with its set of associated linguistic items. We then find all possible 4-tuples $\left(U, H_{1}, H_{2}, L\right)$ where

- $U$ is an utterance.

- $H_{1}$ and $H_{2}$ are hypotheses for $U$, exactly one of which is correct.

- $L$ is a linguistic item of type $T$ that is associated with exactly one of $\mathrm{H}_{1}$ and $\mathrm{H}_{2}$.

If $L$ occurs in the correct hypothesis of the pair $\left(H_{1}, H_{2}\right)$, we call this a "good" occurrence of $L$; otherwise, it is a "bad" one. Counting occurrences over the whole set, we let $g$ be the total number of good occurrences of $L$, and $b$ be the total number of bad occurrences. The discrimination score of type $T$ for $L, d_{T}(L)$, is then defined as a function $d(g, b)$. It seems sensible to demand that $d(g, b)$ has the following properties:

$$
\begin{aligned}
& \text { - } d(g, b)>0 \text { if } g>b \\
& \text { - } d(g, b)=-d(b, g) \text { (and hence } d(g, b)=0 \text { if } g=b \text { ) }
\end{aligned}
$$

\footnotetext{
${ }^{1}$ A summary can also be found in [11].
}

$$
\text { - } d\left(g_{1}, b\right)>d\left(g_{2}, b\right) \text { if } g_{1}>g_{2}
$$

We have experimented with a number of possible such functions, the best one appearing to be the following:

$$
d(g, b)=\left\{\begin{array}{ccc}
\log _{2}(2(g+1) /(g+b+2)) & \text { if } & g<b \\
0 & \text { if } & g=b \\
-\log _{2}(2(b+1) /(g+b+2)) & \text { if } & g>b
\end{array}\right.
$$

This formula is a symmetric, logarithmic transform of the function $(g+1) /(g+b+2)$, which is the expected a posteriori probability that a new $\left(U, H_{1}, H_{2}, L\right)$ 4-tuple will be a good occurrence, assuming that, prior to the quantities $g$ and $b$ being known, this probability has a uniform $a$ priori distribution on the interval $[0,1]$.

One serious problem with corpus-based measures like discrimination functions is data sparseness; for this reason, it will often be advantageous to replace the raw linguistic items $L$ with equivalence classes of such items, to smooth the data. We will discuss this further in Section 3.2.

\section{EXPERIMENTS}

Our experiments tested the general methods that we have outlined.

\subsection{Experimental Set-up}

The experiments were run on the 1001-utterance subset of the ATIS corpus used for the December 1993 evaluations, which was previously unseen data for the purposes of the experiments. The corpus, originally supplied as waveforms, was processed into N-best lists by the DECIPHER ${ }^{T M}$ recognizer. The recognizer used a class bigram language model. Each Nbest hypothesis received a numerical plausibility score; only the top 10 hypotheses were retained. The 1-best sentence error rate was about $34 \%$, the 5 -best error rate (i.e., the frequency with which the correct hypothesis was not in the top 5) about $19 \%$, and the 10 -best error rate about $16 \%$. Linguistic processing was performed using a version of the Core Language Engine (CLE) customized to the ATIS domain, which was developed under the SRI-SICS-Telia Research Spoken Language Translator project $[1,11,12]$. The CLE normally assigns a hypothesis several different possible linguistic analyses, scoring each one with a plausibility measure. The plausibility measure is highly optimized [3], and for the ATIS domain has an error rate of about $5 \%$. Only the most plausible linguistic analysis was used.

The general CLE grammar was specialized to the domain using the Explanation-Based Learning (EBL) algorithm [13] and the resulting grammar parsed using an LR parser [14], giving a decrease in analysis time, compared to the normal CLE left-corner parser, of about an order of magnitude. This made it possible to impose moderately realistic resource limits: linguistic analysis was allowed a maximum of $12 \mathrm{CPU}$ seconds per hypothesis, running SICStus Prolog on a Sun SPARCstation $10 / 41^{2}$. Analysis that overran the time limit was cut off, and corresponding data replaced by null values. Approximately $1.2 \%$ of all hypotheses timed out during

\footnotetext{
${ }^{2}$ All product names mentioned in this paper are the trademark of their respective holder.
} 
linguistic analysis; the average analysis time required per hypothesis was 2.1 seconds.

Experiments were carried out by first dividing the corpus into five approximately equal pools, in such a way that sentences from any given speaker were never assigned to more than one pool $^{3}$. Each pool was then in turn held out as test data, and the other four used as training data. The fact that utterances from the same speaker never occurred both as test and training data turned out to have an important effect on the results, and is discussed in more detail later.

\subsection{Knowledge Sources Used}

The following knowledge sources were used in the experiments:

Recognizer score: The numerical score assigned to each hypothesis by the DECIPHER ${ }^{T M}$ recognizer. This is typically a large negative integer.

In coverage: Whether or not the CLE assigned the hypothesis a linguistic analysis ( 1 or 0 ).

Unlikely grammar construction: 1 if the most plausible linguistic analysis assigned to the hypothesis by the CLE was "unlikely", 0 otherwise. In these experiments, the only analyses tagged as "unlikely" are ones in which the main verb is a form of be, and there is a number mismatch between subject and predicate-for example, "what is the fares?".

Class N-gram discriminants (four distinct knowledge sources): Discrimination scores for 1-, 2-, 3- and 4-grams of classes of surface linguistic items. The class N-grams are extracted after some surface words are grouped into multi-word phrases, and some common words and groups are replaced with classes; the dummy words ${ }^{*} S T A R T{ }^{*}$ and ${ }^{*} E N D^{*}$ are also added to the beginning and end of the list, respectively. Thus, for example, the utterance one way flights to $d f w$ would, after this phase of processing, be ${ }^{*} S T A R T^{*}$ flight_type_adj flights to airport_name ${ }^{*} E N D^{*}$.

Grammar rule discriminants: Discrimination scores for the grammar rules used in the most plausible linguistic analysis of the hypothesis, if there was one.

Semantic triple discriminants:

Discrimination scores for "semantic triples" in the most plausible linguistic analysis of the hypothesis, if there was one. A semantic triple is of the form (Headi, Rel, Head $_{2}$, where Head and $\mathrm{Head}_{2}$ are head-words of phrases, and Rel is a grammatical relation obtaining between them. Typical values for Rel are "subject" or "object", when $\mathrm{Head}_{1}$ is a verb and $H e a d_{2}$ the head-word of one of its arguments; alternatively, Rel can be a preposition, if the relation is a PP modification of an NP or VP. There are also some other possibilities (cf. [3]).

\footnotetext{
${ }^{3}$ We would like to thank Bob Moore for suggesting this idea.
}

\begin{tabular}{|l|c|c|c|c|}
\hline & \multicolumn{4}{|c|}{ Max. length (words) } \\
\hline Preference method & 8 & 12 & 16 & $\infty$ \\
\hline 1-best & 28.3 & 30.4 & 31.9 & 33.7 \\
\hline Highest-in-coverage & 26.3 & 27.4 & 30.1 & 32.2 \\
\hline N-gram/highest-in-coverage & 26.1 & 27.1 & 29.9 & 31.7 \\
\hline Recognizer+N-gram & 25.3 & 27.8 & 29.7 & 31.6 \\
\hline Recognizer+linguistic KSs & 23.3 & 24.8 & 27.9 & 30.0 \\
\hline All available KSs & 23.5 & 25.4 & 28.1 & 29.9 \\
\hline Lowest WE in 10-best & 12.6 & 13.2 & 14.5 & 15.8 \\
\hline \# utterances in 10-best & 442 & 710 & 800 & 843 \\
\hline \# utterances & 506 & 818 & 936 & 1001 \\
\hline
\end{tabular}

Table 1: 10-best sentence error rates

The knowledge sources naturally fall into three groups. The first is the singleton consisting of the "recognizer score" KS; the second contains the four class $\mathrm{N}$-gram discriminant $\mathrm{KSs}$; the third consists of the remaining "linguistic" KSs. The method of [3] was used to calculate near-optimal weights for three combinations of KSs:

1. Recognizer score + class $\mathrm{N}$-gram discriminant KSs

2. Recognizer score + linguistic KSs

3. All available $\mathrm{KSs}$

To facilitate comparison, some other methods were tested as well. Two variants of the highest-in-coverage method provided a lower limit: the "straight" method, and one in which the hypotheses were first rescored using the optimized combination of recognizer score and $\mathrm{N}$-gram discriminant KSs. This is marked in the tables as "N-gram/highest-incoverage", and is roughly the strategy described in [6]. An upper limit was set by a method that selected the hypothesis in the list with the lower number of insertions, deletions and substitutions. This is marked as "lowest WE in 10-best".

\subsection{Results}

Table 1 shows the sentence error rates for different preference methods and utterance lengths, using 10-best lists; Table 2 shows the word error rates for each method on the full set. The absolute decrease in the sentence error rate between $1-$ best and optimized 10-best with all $\mathrm{KSs}$ is from $33.7 \%$ to $29.9 \%$, a proportional decrease of $11 \%$. This is nearly exactly the same as the improvement measured when the lists were rescored using a class trigram model, though it should be stressed that the present experiments used far less training data. The word error rate decreased from $7.5 \%$ to $6.4 \%$, a $13 \%$ proportional decrease. Here, however, the trigram model performed significantly better, and achieved a reduction of $22 \%$.

It is apparent that nearly all of the improvement is coming from the linguistic KSs; the difference between the lines "recognizer + linguistic KSs" and "all available KSs" is not significant. Closer inspection of the results also shows that the improvement, when evaluated in the context of the spoken language translation task, is rather greater than Table 1 


\begin{tabular}{|l|c|}
\hline Preference method & $\begin{array}{c}\text { Word Error } \\
(\%)\end{array}$ \\
\hline \hline 1-best & 7.4 \\
\hline Highest-in-coverage & 6.9 \\
\hline Recognizer+N-gram KSs & 6.8 \\
\hline N-gram/highest-in-coverage & 6.7 \\
\hline Recognizer+linguistic KSs & 6.5 \\
\hline All available KSs & 6.4 \\
\hline Lowest WE in 10-best & 3.0 \\
\hline
\end{tabular}

Table 2: 10-best word error rates

would appear to indicate. Since the linguistic KSs only look at the abstract semantic analyses of the hypotheses, they often tend to pick harmless syntactic variants of the reference sentence; for example all of the can be substituted for all the or what are ... for which are .... When syntactic variants of this kind are scored as correct, the figures are as shown in Table 3. The improvement in sentence error rate on this method of evaluation is from $28.8 \%$ to $22.8 \%$, a proportional decrease of $21 \%$. On either type of evaluation, the difference between "all available $\mathrm{KSs}$ " and any other method except "recognizer + linguistic $\mathrm{KSs}$ " is significant at the $5 \%$ level according to the McNemar sign test [10].

One point of experimental method is interesting enough to be worth a diversion. In earlier experiments, reported in the notebook version of this paper, we had not separated the data in such a way as to ensure that the speakers of the utterances in the test and training data were always disjoint. This led to results that were both better and also qualitatively different; the N-gram KSs made a much larger contribution, and appeared to dominate the linguistic KSs. This presumably shows that there are strong surface uniformities between utterances from at least some of the speakers, which the N-gram KSs can capture more easily than the linguistic ones. It is possible that the effect is an artifact of the data-collection methods, and is wholly or partially caused by users who repeat queries after system misrecognitions.

For a total of 88 utterances, there was some acceptable 10best hypothesis, but the hypothesis chosen by the method

\begin{tabular}{|l|c|c|c|c|}
\hline & \multicolumn{4}{|c|}{ Max. length (words) } \\
\hline Preference method & 8 & 12 & 16 & $\infty$ \\
\hline \hline 1-best & 24.3 & 26.0 & 27.5 & 28.8 \\
\hline Highest-in-coverage & 20.4 & 21.5 & 23.7 & 25.3 \\
\hline Recognizer+N-gram KSs & 20.4 & 22.5 & 23.8 & 25.2 \\
\hline N-gram/highest-in-coverage & 19.0 & 20.5 & 22.6 & 24.1 \\
\hline Recognizer+linguistic KSs & 17.6 & 19.6 & 21.7 & 23.5 \\
\hline All available KSs & 17.6 & 19.6 & 21.5 & 22.8 \\
\hline Lowest WE in 10-best & 11.3 & 12.0 & 13.0 & 14.0 \\
\hline \# utterances & 506 & 818 & 936 & 1001 \\
\hline
\end{tabular}

Table 3: 10-best sentence error rates counting acceptable variants as successes

\begin{tabular}{|l|r|}
\hline Apparently impossible & 14 \\
\hline Coverage problems & 44 \\
\hline Clear preference failure & 21 \\
\hline Uncertain & 9 \\
\hline
\end{tabular}

Table 4: Causes of N-best preference failure

that made use of all available KSs was unacceptable. To get a more detailed picture of where the preference methods might be improved, we inspected these utterances and categorized them into different apparent causes of failure. Four main classes of failure were considered:

Apparently impossible: There is no apparent reason to prefer the correct hypothesis to the one chosen without access to intersentential context or prosody. There were two main subclasses: either some important content word was substituted by an equally plausible alternative (e.g. "Minneapolis" instead of "Indianapolis"), or the utterance was so badly malformed that none of the alternatives seemed plausible.

Coverage problem: The correct hypothesis was not in implemented linguistic coverage, but would probably have been chosen if it had been; alternately, the selected hypothesis was incorrectly classed as being in linguistic coverage, but would probably not have been chosen if it had been correctly classified as ungrammatical.

Clear preference failure: The information needed to make the correct choice appeared intuitively to be present, but had not been exploited.

Uncertain: Other cases.

The results are summarized in Table 4.

At present, the best preference method is in effect able to identify about $40 \%$ of the acceptable new hypotheses produced when going from 1-best to 10-best. (In contrast, the "highest-in-coverage" method finds only about 20\%.) It appears that addressing the problems responsible for the last three failure categories could potentially improve the proportion to something between $70 \%$ and $90 \%$. Of this increase, about two-thirds could probably be achieved by suitable improvements to linguistic coverage, and the rest by other means. It seems plausible that a fairly substantial proportion of the failures not due to coverage problems can be ascribed to the very small quantity of training data used.

\section{CONCLUSIONS}

A simple and uniform architecture combines different knowledge sources to create an N-best preference method. The method can easily absorb new knowledge sources as they become available, and can be automatically trained. It is economical with regard to training material, since it makes use of both correct and incorrect recognizer hypotheses. It is in fact to be noted that over $80 \%$ of the discrimination 
scores are negative, deriving from incorrect hypotheses. The apparent success of the method can perhaps most simply be explained by the fact that it attempts directly to model characteristic mistakes made by the recognizer. These are often idiosyncratic to a particular recognizer (or even to a particular version of a recognizer), and will not necessarily be easy to detect using more standard language models based on information derived from correct utterances only.

We find the initial results described here encouraging, and in the next few months intend to extend them by training on larger amounts of data, refining existing knowledge sources, and adding new ones. In particular, we plan to investigate the possibility of improving the linguistic KSs by using partial linguistic analyses when a full analysis is not available. We are also experimenting with applying our methods to $\mathrm{N}$-best lists that have first been rescored using normal class trigram models. Preliminary results indicate a proportional decrease of about $7 \%$ in the sentence error rate when syntactic variants of the reference sentence are counted as correct; this is significant according to the McNemar test. Only the linguistic KSs appear to contribute. We hope to be able to report these results in more detail at a later date.

\section{ACKNOWLEDGEMENT}

The work we have described was accomplished under contract to Telia Research.

\section{References}

1. Agnäs, M-S., Alshawi, H., Bretan, I., Carter, D.M. Ceder, K., Collins, M., Crouch, R., Digalakis, V., Ekholm, B., Gambäck, B., Kaja, J., Karlgren, J., Lyberg, B., Price, P., Pulman, S., Rayner, M., Samuelsson, C. and Svensson, T., Spoken Language Translator: First Year Report, joint SRI/SICS technical report, 1994.

2. Alshawi, H., The Core Language Engine, Cambridge, Massachusetts: The MIT Press, 1992.

3. Alshawi, H. and Carter, D.M., Training and Scaling Preference Functions for Disambiguation, SRI Technical Report, 1993.

4. Collins, M.J. The Use of Semantic Collocations in Preference Metrics and Word Similarity Measures, M Phil Thesis, Cambridge University, Cambridge, England, 1993.

5. Digalakis, V. and Murveit, H., "Genones: Optimizing the Degree of Tying in a Large Vocabulary HMM Speech Recognizer", Proc. Inter. Conf. on Acoust., Speech and Signal Proc., 1994.

6. Kubala, F., Barry, C., Bates, M., Bobrow, R., Fung, P., Ingria, R., Makhoul, J., Nguyen, L., Schwartz, R. and Stallard, D., "BBN Byblos and Harc February 1992 ATIS Benchmark Results", Proc. DARPA Workshop on Speech and Natural Language, 1992.

7. Murveit, H., Butzberger, J., Digalakis, V. and Weintraub, M., "Large Vocabulary Dictation using SRI's DECIPHER ${ }^{T M}$ Speech Recognition System: Progressive Search Techniques", Proc. Inter. Conf. on Acoust., Speech and Signal Proc., Minneapolis, Minnesota, April 1993.
8. Norton, L.M., Dahl, D.A. and Linebarger, M.C., "Recent Improvements and Benchmark Results for the Paramax ATIS System". Proc. DARPA Workshop on Speech and Natural Language, 1992.

9. Ostendorf, M., et al., "Integration of Diverse Recognition Methodologies Through Reevaluation of N-best Sentence Hypotheses," Proc. DARPA Workshop on Speech and Natural Language, 1991.

10. Powell, F.C., Cambridge Mathematical and Statistical Tables, Cambridge University Press, Cambridge, England, 1976.

11. Rayner, M., Alshawi, H., Bretan, I., Carter, D.M., Digalakis, V., Gambäck, B., Kaja, J., Karlgren, J., Lyberg, B., Price, P., Pulman, S. and Samuelsson, C., "A Speech to Speech Translation System Built From Standard Components". Proc. ARPA workshop on Human Language Technology, 1993

12. Rayner, M., Bretan, I., Carter, D., Collins, M., Digalakis, V., Gambäck, B., Kaja, J., Karlgren, J,, Lyberg, B., Price, P., Pulman S. and Samuelsson, C., "Spoken Language Translation with Mid-90's Technology: A Case Study". Proc. Eurospeech '93, Berlin, 1993.

13. Samuelsson, C. and Rayner, M., "Quantitative Evaluation of Explanation-Based Learning as a Tuning Tool for a Large-Scale Natural Language System". Proc. 12th International Joint Conference on Artificial Intelligence. Sydney, Australia, 1991.

14. Samuelsson, C., Fast Natural Language Parsing Using Explanation-Based Learning, $\mathrm{PhD}$ thesis, Royal Institute of Technology, Stockholm, Sweden, 1994. 